\title{
User Interface Design of E-Learning System for Functionally Illiterate People
}

\author{
Asifur Rahman \\ Graduate School of Information Science and Electrical \\ Engineering, Kyushu University \\ Fukuoka, Japan
}

\author{
Akira Fukuda \\ Faculty of Information Science and Electrical Engineering \\ Kyushu University \\ Fukuoka, Japan
}

\begin{abstract}
Among different type of illiterate people, the print illiterates suffer most from getting crucial information passed around the society. Many print illiterate people are found in the developing countries and in many cases they live in the remote areas working as farmers. These people are deprived of the knowledge generated from the latest scientific researches. This research makes some recommendations related to developing user interface especially suitable for the print illiterate people. In this regard, a user interface is developed based on the recommendations from the previous researchers. The authors find the recommendations insufficient and develop another user interface based on the improvements proposed by the authors. Later both the user interfaces are tested by two different groups of print illiterate people in a remote village in Bangladesh. The test data shows that the proposed improvement contributes significantly to make the user interface more usable to the target population. 13 out of 15 users could complete the assigned task successfully using improved user interface. Whereas only 8 out of 14 users could do the same with the other user interface. Among the successful users, the improved user interface took $26 \%$ less time than that of the other user interface. Finally some recommendations to develop user interface for the functionally illiterate people are made based on the results and observations of this research.
\end{abstract}

\section{Keywords-User Interface; Illiterate people; e-Learning}

\section{INTRODUCTION}

In the backdrop of evolving different technologies, more complex set of illiteracy is being observed in the society. Those include computer illiteracy, media illiteracy, visual illiteracy, etc. $[1,2]$. These illiterate people can read and write on a specific language (usually in their native language), however, cannot respond to the respective technology. Inability of reading and writing is termed as print illiteracy. Most of the people in this world are print illiterate except a few languages. There is another type of illiteracy observed among the people migrated from one country to another and are unable to read and write the local language [3]. These people might be very efficient in their native language, though. These types of migrated people usually fall into the category of print illiterate. It can be summarized that every individual belongs to some groups of illiteracy. The extent of literacy also varies from highly skilled to functionally illiterate.

Among different types of illiterate people, print illiterates are the group who suffers most from getting information passed around the society. The largest group of print illiterate people is found in the developing countries. These illiterate people live both in the urban area and in the remote area. Illiterate people living in the remote area suffer more from getting information than ones living in the urban area. Many researches are conducted to introduce innovative ICT-based services to provide information to the people living in the remote areas in the developing countries [4-5]. The researchers also discussed the constraints and opportunities in details. Many of the researches are found not attaining the planned goals because the concerned users cannot use the system properly and are not happy with the benefits getting from the system [6]. Therefore, the target population, despite its usefulness, abandons the system. This research aims to develop a user interface for the print illiterate people.

It is obvious that the access to knowledge and information is the primary means of empowering the people living in the remote areas. They possess the same potential like that of a literate urban people [7]. They are surviving for generations and fighting against all odds without the help of latest scientific research findings. They live based on the skills and techniques learned from their surroundings. The authors intend to develop a platform for them to accelerate their learning considering existing infrastructural limitations, like lack of electricity supply, poor internet connectivity, print illiteracy, poverty, and so on. The learning process is optimum when it is made personalized [8]. Therefore, the researchers aim to develop an e-Learning system [9] that utilizes multimedia and internet technologies to facilitate the users to access services and resources as and when needed.

The existing digital library and e-Learning systems are using multi-media extensively. The audio was used in some projects as technological means to disseminate information to the illiterate people [10]. Non-computerized systems used audio and video cassettes in this regard [11-13]. These are convincing examples that demonstrate that information can be distributed, accessed and created via appropriate technologies that do not need print literacy. The authors decided to use computerized technology to store videos demonstrating the usage of different skills that can empower people in the remote areas and distribute those through a system that does not require print literacy to use. Therefore, developing a videobased e-learning system using an innovative user interface especially suitable for illiterate people. Use of such user interface that can be used by the illiterate people without any help from others makes the application to be self-operated and low cost. 
Usability of an application depends more on its ease of use for the target population than its features. There are evidences that many high-quality applications were abandoned because the target users could not use them properly. Considering the target population, the print illiterate people, it is important that the user interface is suitable enough for them. Besides, the authors assume that the application would be available in the remote areas and be operated by the users themselves without any help from others. Therefore, the focus was on developing a user interface that an illiterate person can operate independently.

The rest of this paper is organized as follows: Section 2 contains the related works on developing user interface for the illiterate people. The existing works are categorized based on the technologies used. The limitations of those user interfaces and usability in specific situations are also mentioned. Section 3 details the prototype developed in this work. Section 4 contains the primary design of the user interface. It also describes the primary evaluation of the user interface, findings, and observations. Section 5 describes the improvements that the authors proposed on the existing recommendations to make the user interface more effective to the illiterate people. Section 6 details the experiment conducted and analysis of data. Section 7 contains the discussion, and section 8 concludes with a list of future works.

\section{RELATED WORK}

User interface for print illiterate people became a subject of interest for the researchers for quite a long time. Different approaches have already been tested to develop a user interface and improve its usability for the print illiterate people. All these attempts might be categorized as: i) innovative use of text and numbers; ii) Use of extensive graphics and minimum text; and iii) Use of audio in addition to text and graphics.

In the early days of computer application development, command line based user interface was the only means of manmachine interaction. Soon after the computers had more processing capabilities, text-based menu was introduced. Textbased menu was shortly replaced by graphics-based menu. During this time it was not considered that poor illiterate people living in the developing countries would use computers. In the early of 21 st century, when the price of computers came within the range of common people in the developing countries, the governments and the international development agencies took initiatives to develop application for the people living in the rural areas of the developing countries. However, it was always remain as a challenge to make the rural illiterate and semi literate people using those. Ample of evidences were found where many potential software applications were abandoned because the target illiterate and semi literate users could not use those. This observation encouraged the researchers to work extensively to develop suitable user interface for the illiterate people.

Researches have shown that the illiterate people can remember numbers more easily than words. Therefore, some experiments used more numbers to make it easy for the illiterate users to use the applications [14-18]. However, these recommendations based on usage of number against text are more relevant for mobile phone interface and other man- machine interface where high quality multimedia capabilities are not available.

Some other researchers used speech recognition techniques to solve the problem of illiteracy [19-23]. In these applications, the researchers used short word based commands to activate tasks. In India, China, Bangladesh and some African countries, local dialects varies from district to district within the same state. It would be very difficult to train the speech recognition system to work for a considerably large segment of people. However, the systems work satisfactorily with very limited command set used by people from only small number of villages.

In the recent days, some researchers introduced new techniques using the recent improved hardware and software features [24-28]. Researchers have also tested the effect of different types of graphics like sketch, photo, animated cartoon and video. In different situation the result varies significantly. The researchers also recommended some guidelines for developing effective user interface for the illiterate users.

Considering availability of rich hardware features and software platforms the authors choose to use the recommendations for developing the user interface of the intended e-Learning system for the print illiterate people using text, graphics and intelligent use of audio annotation. Details of the design considerations are explained in a separate section.

\section{E-LEARNING SYSTEM PROTOTYPE FOR ILLITERATE PEOPLE}

The authors are intending to build an e-learning system for the illiterate people living in the remote areas of developing countries to distribute knowledge and empowering them. The knowledge includes farming skills, public health, sanitation, child nutrition, maternity care, etc. Technical and non-technical limitations are considered on construction of this system.

The system is organized as shown in Fig. 1. The content server works as the central repository of all data. In addition to the e-Learning system and its related database, database replication management module and DTN module are also available in the content server. The database replication management module ensures that all the local server databases are synchronized properly with the content server periodically. The internet bandwidth in remote areas of developing countries is not suitable yet to transfer bulky multimedia data. Therefore, the bulk data transfer is arranged from content server to the local server over Delay Tolerant Network (DTN) [29]. DTN is a hop-to-hop store and forward data transfer protocol useful in the challenged infrastructure scenario. DTN uses vehicles like Bus, Steamer, Satellites, etc. to transfer data physically from one place to another. In this work, data is transferred from the content server to the local server over internet and DTN using the interim facilities: DTN relay center and DTN node in bus. The local server contains the e-Learning system, database replication management module, and DTN module. The local servers are installed in village information centers (VIC). Here, VIC is a shared ICT service access facility owned by a local entrepreneur, that offer computers and internet connectivity to the villagers to use against money. The details of the DTN 
module, DTN relay center, and DTN node in bus is out of the scope of this paper.

The content server contains a database that stores video contents and related data. The database replication management module decides which content to be replicated in which local server. It is obvious that the central server database is a huge one. Local servers located in different areas do not need to contain each and every content. For example, mango fruit is not cultivated everywhere in Bangladesh. It is very unlikely that a villager, living in a village where mango fruit is not cultivated, will search for a video related to mango cultivation. Therefore, while replicating the database, contents are selected for a local server based on socio-economic, demographic, agricultural, environmental, and other information related to that area. The database replication management module also ensures that all the local servers are synchronized appropriately periodically.

The content server is accessible over internet. The users of this system may be either registered or anonymous. The administrators and the content providers are the registered users. Both the registered and anonymous users can access the system at any time. Anonymous users can only search for and play video contents. Content providers represent organizations those are interested to produce and share the videos to help the remote villagers. A content provider can upload videos and fill up corresponding data at any time over the internet. He/she can also edit or delete his/her own contents.

The villagers visit village information center (VIC) to use the system. They do not need any registration and can only search for and play the videos.

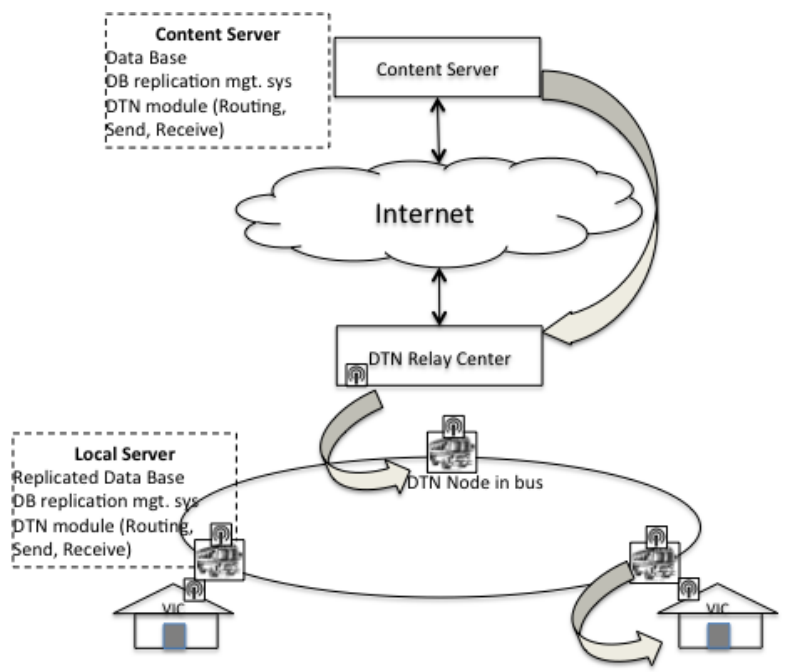

Fig. 1. System Arrangement

\section{A. Features of the system:}

The system maintains a repository of video contents that teaches different skills and techniques useful for the target population. It also contains information about new crops, livestock, etc. The features of the systems are briefly described as follows:

a) User Registration: People who are literate can fill up a form to become a registered user. Then the user input his identification information. The registration process completes with verification and approval from the administrator. The registered users can upload video content, edit, delete data or administer the system based on their granted privilege.

b) Content uploading: The registered users may upload a video content after login the system properly. During uploading the video, the content provider provides information like the category, subcategory, language, country, etc. of the video. The content uploader also need to enter a short description of the video and audio of the text description. The process is made such to ensure accountability against the uploaded contents.

c) Content editing: Content providers and the administrators can use this option to edit information related to any specific content. A content provider can only edit data of the contents he uploaded.

d) Content deleting: Content providers and administrators can use this feature to remove any content from the system. A content provider can only remove the contents he uploaded.

e) System configuration: The configuration privilege is only granted to the administrator. Configuration includes content server configuration, data replication criteria setting for different local servers, parameter setting for data transportation from content server to local server and so on.

f) Content searching: This feature can be used by both registered and anonymous users. The literate users can search using a text based user interface and the illiterate users can search using a different user interface especially suitable for illiterate people.

g) Content playing: The user can search the desired video using the search option. Then he/she can play the video to watch using content playing feature.

Most of these features are for administrators or content providers who are literate and possess high IT aptitude. Considering the scope of this paper, Content Searching, and Content Playing should be discussed extensively.

$\begin{aligned} & \text { Use case name: } \\ & \text { Summary: } \\ & \text { Takes input from the user and search for } \\ & \text { matching video contents from the } \\ & \text { database and list. }\end{aligned}$
Description:
1. User specifies to search contents.
2. User specifies the category, subcategory, language,
country, and full or part of the title of the contents to be
part of the list. User may input all or some of the criteria
mentioned above.
3. The system searches for the matching contents.
4. The system lists the matching contents.

Fig. 2. Content Searching use case

\section{B. Content Searching:}

Content searching is the most frequently used feature. Administrators and the content providers use it to edit or delete contents. They may also use it to play contents. Anonymous users always use it to find out the desired content before playing it. Fig. 2 shows the use case. 


\section{Content Playing:}

After the user finds the desired content using the content searching feature, he/she will use content playing feature for watching. It is another frequently used feature. Fig. 3 contains the use case related to content playing feature.

Use case name: Play content
Summary:
Description:
1. User specifies the video content that he/she wants to play
2. The system starts playing the video content
3. User watches the video content
4. The system finishes playing the video content, moves the
playing position to the beginning of the video content,
and returns into the pause mode

Fig. 3. Content Playing use case

\section{PRIMARY DESIGN OF USER INTERFACE}

For the purpose of developing the user interface, the authors followed the recommendations made by the previous researchers for developing user interface of similar applications [24-27]. The recommendations are:

a) Minimizing the need of hierarchical navigation. It requires higher level of aptitude to remember the combinations that emerge after a few steps. The situation gets worse when the number of branches in each level becomes higher.

b) Maintaining natural workflow as much as possible. It reduces the load of learning new concept.

c) Use of graphics where users need to select an item. Text does not bear any meaning to an illiterate person. Most of the cases, a picture explains the meaning more clearly than words.

d) Use of Audio annotation to clarify confusing situation. There are situations when it becomes very difficult to develop two distinctly different pictures for two very similar situations. For example, in Fig. 4(a) is a photo of pests and Fig. $4(b)$ is a photo of insects. Both the photos are collected from Internet. These photos are too similar to differentiate as two different categories pest and insect. Audio annotation in such cases helps significantly.

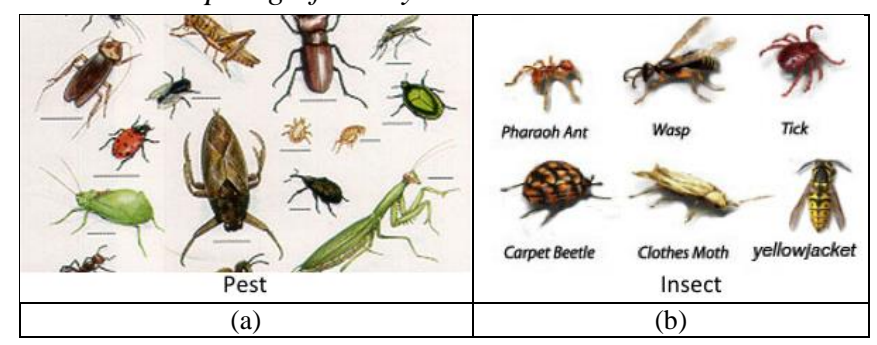

Fig. 4. Photos for categories pest and insect

e-Health was used as an application domain to test the design principals in the aforementioned work. In this work, the authors followed the same recommendations to design the user interface for e-Learning system.

The authors developed a user interface for the illiterate people following the aforementioned recommendations. Fig. 5 shows the user interface, which is refined from the use case description of Content Searching use case. The details of the user interface is described below:
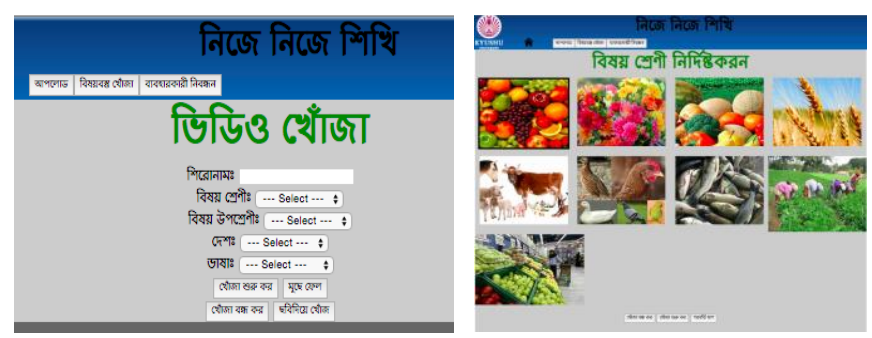

(a)

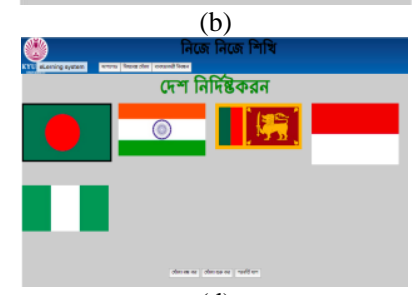

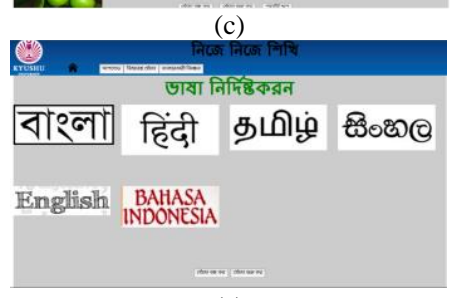

(e)

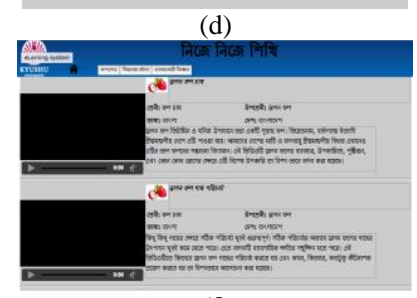

(f)
Fig. 5. User interface of Content Searching use case: Fig. (a) shows the user interface for literate users; (b) shows the categories an illiterate user can select: fruit, flower, vegetable, etc.; (c) shows the sub categories an illiterate user can select: mango, jack fruit, orange, strawberry, etc., which are the subcategories of the category fruit; (d) shows the countries an illiterate user can select: Bangladesh, India, Sri Lanka, Indonesia, and Nigeria; (e) shows the languages an illiterate user can select: Bengali, Hindi, Tamil, Sinhala, English, and Bahasa Indonesia; (f) shows the list of contents match the criteria specified in the previous steps

1) The user specifies to search for contents by clicking the search content button available in the header of all the webpages. This search content button is active in all the webpages. The webpage shown in Fig. 5(a) appears.

2) The user specifies the category, subcategory, language, country, and full or part of the title of the contents to be part of the list by selecting one from the list box or filling the text field in the webpage shown in Fig. 5(a). For the print illiterate user, all the text is supplemented by audio annotation. Moreover, when the user makes the mouse cursor hover on a text filed or a button for two or more seconds, the webpage plays the audio annotation explaining function and purpose of the underlying object.

Since it is full of text and not suitable for illiterate users, another webpage of the same features for the illiterate people is also provided. By clicking the bottom right button of the webpage shown in Fig. 5(a), another version of the webpage shown in Fig. 5(b) appears.

The webpage is graphics based and more suitable for the print illiterate people. The webpage shows pictures of fruit, flower, vegetable, etc., which represent categories the user can select. The user can select a category by clicking on the photo. 
The selected category would be marked with a black border. Moreover, if the user makes the mouse cursor hover over any picture, the webpage tells the name of the category in voice. Similarly, the print illiterate people can select a subcategory, a country, and a language in the webpages shown in Fig. 5(c), (d), and (e), respectively, which appear subsequently.

Moreover, the system also explains the purpose of any button if the user makes the mouse cursor hover over it.

3) The system searches the database for the matching contents.

4) The system lists the matching contents in the webpage shown in Fig. 5(f). The print illiterate user can identify exactly which one of the matching contents the user wants to play by making the mouse cursor hover over the content description and listening it in voice.

After the user specifically identifies which content the user wants to play, then he/she would click on the video to start playing. This would start execution of Content Playing use case and the user interface would look like Fig. 6.

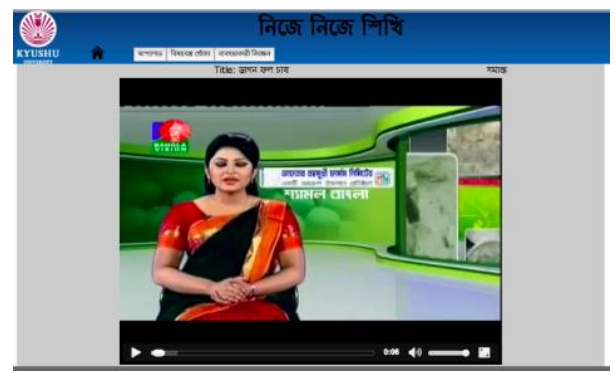

Fig. 6. User interface for Content Playing use case

Like the previous user interface page, all the text and buttons of this page are also supplemented by audio annotation. The user would put the cursor on different text and buttons to know the purpose and use of the same if necessary.

After developing the user interface the performance was tested with a trial evaluation. Some Indonesian and Japanese students, who do not understand Bangla language, used the system as illiterate users. Although the real system uses audio annotation in Bangla language, English and Japanese annotation were used for Indonesian and Japanese students respectively, so that the users can understand the audio annotation. Each user was given some time to learn the system and then was asked to find a video based on a description. It was expected that the users would use the system independently. Every user could find the desired video. However, the number of wrong attempts in different pages was quite high.

Following are the observations of the trial performance test:

a) All the users are familiar with use of computers. Therefore, they are very conversant to the user interface and the workflow concept. However, they could not understand the text in Bangla Language. Still it took long time for them to find out which button to choose at what stage.

b) Users kept listening to the audio annotation of different elements until the appropriate option was found. c) Sometimes, the users cannot concentrate on the audio descriptions because the number of prospective buttons is quite large. That leaded to errors.

d) Users feel discouraged to try too many options to find the correct button or text.

The authors identified following limitations of the user interface developed:

a) Users get frustrated if there are many options available to choose in a user interface to do different tasks and users repeatedly make wrong guess. Therefore, available or probable options for the users are to be minimized.

b) Sometimes users cannot recall from where to start and the purpose of the page. Because he/she cannot read the interface texts or button labels. Therefore, a proactive briefing might be useful for the users.

\section{IMPROVED DESIGN OF USER INTERFACE}

Based on the aforementioned observations and findings, following improvements were made in the user interface design:

a) To minimize the number of options to choose, the authors intended to guide the users to choose from a limited number of most probable options. Among different buttons available in the page, the authors identified the most likely to be used buttons to go to the next options. There are also some text information that the majority users need to know. Those buttons and texts are made blinking to attract attention from the users.

b) To help users recall the purpose of the elements of each page, a proactive audio description of each page was added. The description was played after the page is loaded. Users may listen to the description before taking action in case he/she forgot the purpose of that specific page.

Fig. 7 shows the user interface for the Content Searching use case after the aforementioned improvements. In Fig. 7(a) the text-based user interface is for the literate users and they don't need any assistance from the user interface. Illiterate users may choose one from two options: i) they will move forward to search with photos; ii) they will abandon the search. Therefore, the corresponding two buttons are made blinking. To listen to the purpose of each of these buttons, users may hover over the buttons and choose the appropriate one. There are 13 dropdown list, textboxes and buttons available in the page. This effort indicates that these two blinking buttons are the most probable option for an illiterate user to choose. Similarly in all the pages, some buttons or textboxes are made blinking, so that the illiterate users can understand that these are the probable buttons or textboxes to choose at this stage.

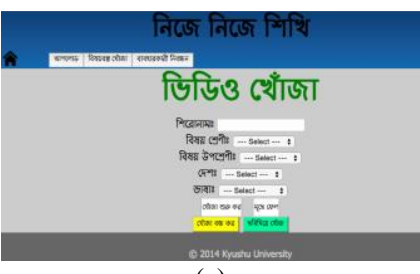

(a)

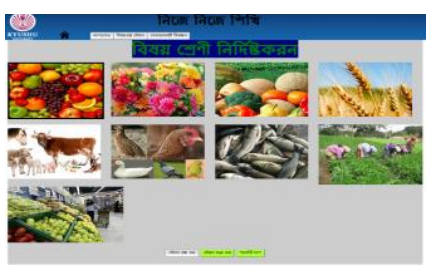

(b) 


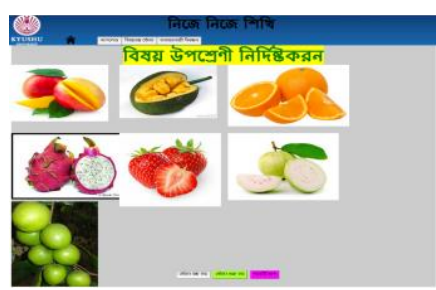

(c)

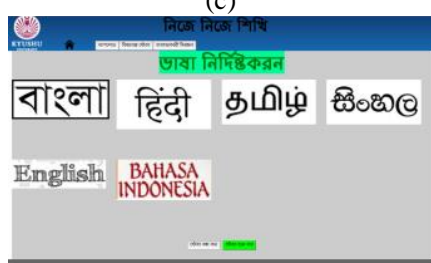

(e)

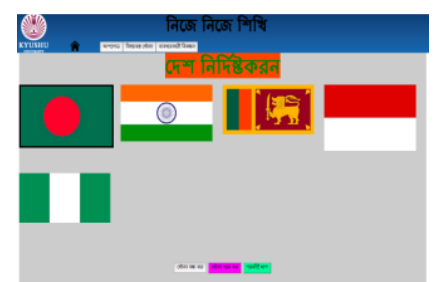

(d)

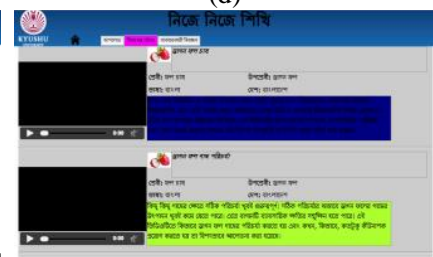

(f)
Fig. 7. User interface for Content Searching use case after improvement. The figures are same as Fig. 7 (a)-(f) except in each page few buttons and textboxes are blinking

As mentioned earlier the proposed second improvement, proactive audio description for each page is added. The description is played immediately after the page is loaded. The purpose of the page, action invoked by clicking each blinking button, information content in each blinking textbox is explained in the audio description. If the illiterate users forget the purpose of the buttons and textboxes available in the page, this proactive description allows them to recall and start using the page.

The aforementioned changes are made in every page. The new user interface for the use case Content Playing is shown in Fig. 8. While playing content, the user may want to finish watching the video and go back to the previous list or start searching another content. Therefore, two buttons corresponding to the mentioned actions are made blinking.

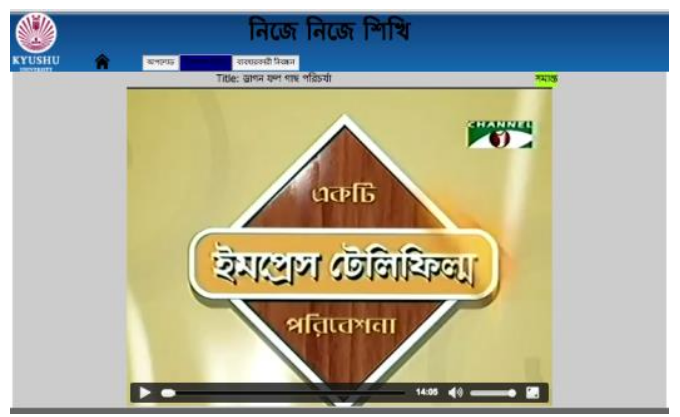

Fig. 8. User interface for Content Playing use case after improvement

\section{EXPERIMENT AND DATA ANALYSIS}

Purpose of this work is to develop an effective user interface for the illiterate people. The authors identified some limitations of the existing proposals for the same and improved the existing proposals to develop a new user interface. Finally compared the performance of two versions of user interface, one developed based on the existing proposal and another developed based on the proposed improvements.

\section{A. Evaluation Process:}

The first version, (henceforth referred as UI-1), which is developed based on the existing recommendations, was evaluated by a group of 14 functionally illiterate people in a village in Bangladesh and the improved version, (henceforth referred as UI-2), was evaluated by another group of 15 functionally illiterate people from the same village. The participants of both the groups are selected randomly among the male residents of the same village. The age of the participants were uniformly distributed among different age groups. All of them are functionally illiterate. Some of them can read and write though. The participants have experience of using mobile phone. Although some of them saw computers, however, none of them used computer. The workflow of the evaluation process is as follows:

1) The evaluation process starts with a detailed description of the system to the user. The user also shown how to use the system. The purpose of evaluation, the role of the user in the process is also described in details. It is categorically mentioned to the user that the purpose of this evaluation is to measure the usefulness of the user interface only. The personal data, like name, age, educational qualification, etc. are also collected during this time.

2) The user is allowed to practice using the system for about an hour, so that they can learn how to use it. During this practice session, they were guided as and when needed.

3) The user is given a list of tasks to perform. The example of a task might be "find a video that describes the scientific process of raising a cow for milk production." The user performs the tasks and his activity during this period is recorded. Later this recorded video clip is used to collect data like time to complete the task, mistakes during search, etc.

4) Oral feedback about the system is collected from the user.

\section{B. Results:}

The participants were given a list of tasks to complete during the evaluation. Example of a task can be "find a video that describes the scientific process of raising a cow for milk production." The successful completion of the aforementioned task means that the participant could find the correct video. While performing the task, a participant might find a wrong video or after repeated choice of wrong steps a participant may abandon the search and starts from the beginning.

a) Successful completion: 14 participants used "UI-1" and 15 participants used "UI-2". The performance of the participants of "UI-1" and "UI-2" is shown in Table I. The data shows that 8 out of 14 participants could complete the task using "UI-1". On the other hand, 13 out of 15 participants could complete the task using "UI-2". Therefore, more people can use "UI-2" successfully than "UI-1".

TABLE I. NumBer OF PARTICIPANTS COMPLETED TASKS USING BOTH THE USER INTERFACES

\begin{tabular}{|l|l|l|}
\hline & UI-1 & UI-2 \\
\hline Total Participants & 14 & 15 \\
\hline Failed to complete & 6 & 2 \\
\hline Completed without hints & 6 & 9 \\
\hline Completed with hints & 2 & 4 \\
\hline
\end{tabular}


b) Completion time comparison: The number of tasks attempted and completed by the participants is not same for all participants. The number of attempts depended on interest of the participant on the system and time spent on learning the system. Most of the participants could complete at least one task successfully. Time spent for the unsuccessful attempts is ignored during calculating the average completion time. For the participants, who completed multiple tasks, the authors considered average time of completion as their completion time. Average time of completion for all participants using "UI-1" is 6:32 minutes whereas average time of completion for all participants using "UI-2" is 5:10 minutes. Therefore, it can be concluded from the data of this experiment that, in similar situation a participant can use "UI-2" $26 \%$ faster than "UI-1".

c) Age wise performance: It is known that younger people can respond to the new technology faster and older people suffer from techno phobia, therefore, fails to use new technology. However, in this work it is found that people from different age groups could use the system similarly. The participants were selected almost equally among different age groups. Table II shows the number of participants became successful and unsuccessful in different age groups. The data shows that combining all participants of "UI-1" and "UI-2" every age group has 2 unsuccessful persons.

TABLE II. SUCCESSFUL AND UNSUCCESSFUL PARTICIPANTS IN DIFFERENT AGE GROUP

\begin{tabular}{|l|l|l|}
\hline Age group & Successful & Unsuccessful \\
\hline Under 20 & 5 & 2 \\
\hline Under 30 & 5 & 2 \\
\hline Under 45 & 6 & 2 \\
\hline Rest & 5 & 2 \\
\hline
\end{tabular}

VII. DISCUSSION

The analysis of the data collected from the experiment shows that:

1) More people can use "UI-2" successfully than "UI-1".

2) Users can find the desired video faster in "UI-2".

3) The user interface is equally useful to all different age groups.

It indicates that the recommendations made by previous researchers for similar applications are also applicable in the present scenario. However, the proposed improvements affect significantly in the situation under consideration of this research.

\section{A. Recommendations:}

Based on the observations and data analysis, following design principals can be recommend for the UI development for the functionally illiterate people:

1) Use of images and graphics instead of text: People, who cannot read, can understand photos. It is important that the photo emphasizes a specific message only. The background or other object present in the photo does not get prominence. It is found in the previous works that illiterate people can understand sketch better than photos. The background and other objects available in the photo drags their attraction that makes them confused sometimes. Although photos were used in this experiment, users were not confused. The photos used in the user interface are selected carefully to emphasize only one message.

2) Use of audio annotation: In many cases it becomes very difficult to represent two very close subjects with two distinct graphics images. Therefore, it requires using audio annotation in addition to graphics representation. In this research, audio annotation during hover action found the most effective.

3) Adding context sensitive suggestions to the users: Guided and controlled users action reduces errors and mistakes. The illiterate users should always be guided to the possible actions for a specific situation. However, the designer should be careful to select most likely options for a specific situation. Too many suggestions may confuse the users.

4) Proactive briefing: In this research, it is found that a proactive description of the features and possible use of different active elements is very effective for the users to select the correct button or option. Users with less orientation to the technology cannot remember all the steps accurately. Besides, due to lack of experience they cannot make good guess. Therefore, early briefing would help them to make fewer mistakes. Long verbal description might be avoided by some graphics based animated description in order to make it interesting.

\section{B. Observations:}

After completion of using the system the users were requested for their comments about the user interface and the system. This session was their first experience of using computers. Therefore, they could not articulate their opinion very clearly. The authors are listing their observations acquired through the whole activity:

1) Orientation to computer usage is an essential part of using any computer-based system. Whatever smart user interface is developed, users cannot use it unless they learn basic computer usage. Therefore, the users need basic computer training for two weeks or more.

2) Age was not a factor for learning and using the system. Appropriate use of graphics, audio annotation, and proactive and innovative guidance were the reasons behind the success.

3) Schooling does not influence learning process. There are people who have gone higher classes in the school but cannot respond well. Some people who can read and write a little still failed to ensure good learning of the system.

4) All participants could understand the audio instruction but it was difficult for a first time user to remember the workflow after listening the audio instruction.

5) Audio annotation is essential during training period. However, it should be brief and to the point. Long description and repetition should be avoided. After the users get used to the system then long description of each page would be very annoying. 


\section{6) Animated guidance used in "UI-2" is very effective.}

\section{CONCLUSION AND FUTURE WORKS}

This research demonstrates that it is possible to develop a user interface that allows functionally illiterate people to learn and use a system without any help from others. This success would encourage governments and development organizations to introduce more and more new systems to help the people living in the remote areas to improve their quality of living. A web-based system would further allow the service provider to maintain and monitor the system remotely from the cities.

This study was conducted for a computer-based system. However, the results and recommendations can also be used in other situations for man-machine interface. Consider a situation when a person is moved to another country where the local language is different. In that case, that person becomes a newly illiterate person. While this person needs to use vending machines, he faces the same situation like that of an illiterate person faces in the remote areas with a computer system. The recommendation can be used to develop a system that is easily usable.

This research put some recommendation that demands more resources to the system. For advanced and complex systems, the additional resources could become very exhaustive. In future, the authors would extend the features of the e-Learning system to make it more useful to the users. and would test with the real users for a considerable period of time. This would allow to study the performance of the proposed user interface more accurately. Finally, the authors would like to come to a conclusion for the optimum level of details for the audio annotation, proactive guidance, etc.

\section{REFERENCES}

[1] D. Bawden, "Progress in Documentation, Information and Digital Literacy: a Review of Concepts", in Journal of Documentation, vol. 57, no. 2 pp 218-259, 2001.

[2] M. Walton, G. Marsden and V. Vukovic, "'Visual Literacy' as challenge to the Internationalization of Interface: A study of South African Student Web Users" in Proceedings of the CHI-SA Conference 2001, Pretoria, South Africa, pp. 530-531, 2001

[3] Y. L. Theng, N. Mohd Nasir, H. Thimbleby, G. Buchanan, M. Jones, et al, "Children as Design Partners and Testers for a Children's Digital Library," in Proceedings of the 4th ECDL Conference, Springer Verlag, pp. 249-258, 2000

[4] T. Almarabeh and A. AbuAli, "A General Framework for EGovernment: Definition Maturity Challenges, Opportunities, and Success," in European Journal of Scientific Research, Vol. 39 No. 1 pp. 29-42, EuroJournals Publishing, Inc. 2010.

[5] G. Sharma, X. Bao and L. Peng, "Public Participation and Ethical Issues on E-governance: A Study Perspective in Nepal" in Electronic Journal of e-Government Volume 12 Issue 1, pp82-96, 2014.

[6] R. Heeks, "Information systems and developing countries: Failure, success, and local improvisations." In The information society vol. 18, no. 2, pp. 101-112, 2002.

[7] N. P. Sullivan, "You Can Hear Me Now: How Microloans and Cell Phones are Connecting the World's Poor To the Global Economy", John Wiley \& Sons, 2007

[8] M. Gell-Mann, "A commentary to R Schank. The third culture; beyond the scientific revolution." New York: Touchtone Books, pp. 167-180, 1996.

[9] EC [European Commission], Communication from the commission to the council and the European parliament: the e-Learning action plan, Brussels, 28.3, 2001
[10] A. Mutshewa, "Disseminating Environmental Information in Rural Botswana: the Case of Nata Village," in Information Development vol. 15, no. 2 pp. 96-102, 1999

[11] A. P. du Plooy, "Community Information for the Functionally Illiterate in South Africa by Means of Audio-Cassettes," in Mousaion vol. 6, no. 1 pp. 2-20, 1988.

[12] M. Rahnema, "The Sound Library, a Simple but Revolutionary Tool for Development," in Unesco Journal of Information Science, Librarianship and Archives Administration vol. 4, no. 3 pp. 151-158 1982.

[13] P. Manuel, "Cassette Culture: Popular Music and Technology in North India," University of Chicago Press 1993.

[14] Z. Lalji and J. Good, "Designing new technology for illiterate population: A study in mobile phone interface design", in Interecting with compiters vol. 20, no. 6, pp. 574-586, 2008.

[15] I. Medhi, S. Patnaik, E. Brunskill, S. N. N. Gautama, W. Thies et al. "Designing Mobile Interface for Novice and Low-Literate Users", in ACM Transactions on Computer-Human Interaction, Vol. 18, No. 1, Article 2, 2011

[16] T. S. Parikh, "HISAAB: An Experiment in Numerical Interfaces", Media Lab Asia Panel Discussion, Baramati Initiative on ICT and Development., MIT, 2002

[17] T. S. Parikh, K. Ghosh, and A. L. Chavan, "Design Considerations for a Financial Management System for Rural, Semi-literate Users," in the procedings of $\mathrm{CHI}$ '03 Extended Abstracts on Human Factors in Comouter Systems, Ft. Lauderdale, Florida, USA, pp. 824-825, April 2003

[18] T. S. Parikh, K. Ghosh, and A. L. Chavan, "Design Studies for a Financial Management System for Micro-credit Groups in Rural India," in the Procedings of the 2003 conference on Universal Usability,Vancouver, British Columbia, Canada, pp. 15-22, November 2003.

[19] M. Plauche and M. Prabaker, "Tamil market: a spoken dialog system for rural India" in the proceeding CHI '06 Extended Abstracts on Human Factors in Computing Systems Pages, pp. 1619-1624, 2006.

[20] M. Plauché, U. Nallasamy, J. Pal, C. Wooters and D. Ramachandran, "Speech Recognition for Illiterate Access to Information and Technology," in the procedings of International Conference on Information and Communication Technologies and Development, 2006, pp. 83-92, 2006.

[21] A. Lerer, M. Ward and S. Amarasinghe, "Evaluation of IVR Data Collection UIs for Untrained Rural Users," in the Proceedings of the First ACM Symposium on Computing for Development, December, 2010, London, United Kingdom, p. 2, 2010.

[22] K. Bali, S. Sitaram, S. Cuendet, and I. Medhi, "A hindi speech recognizer for an agricultural video search application," in the procedings of the 3rd ACM Symposium on Computing for Development, January 2013, P. 5, 2013.

[23] S. Cuendet, I. Medhi, K. Bali, E. Cuttrell, "VideoKheti: Making Video Content Accessible to Low-Literate and Novice Users," In the procedings of the SIGCHI conference on Human Factors in Computing Systems, 2013, pp 2833-2842, 2013

[24] I. Medhi, M. Lakshmanan, K. Toyama and E. Cutrell, "Some Evidence for the Impact of Limited Education on Hierarchical User Interface Navigation," in the procedings of the SIGCHI conference on Human Factors in Computer Systems, 2013, pp. 2813-2822, 2013.

[25] I. Medhi, and R. Kuriyan, "Text-Free UI: Prospects for SocialInclusion," in the procedings of International Conference on Social Implications of Computers in Developing Countries. Brazil, 2007

[26] I. Medhi, B. Pitti, and K. Toyama, "Text-Free User Interfaces for Employment Search," in the Procedings of Asian Applied Computing Conference, Nepal, 2005.

[27] I. Medhi, A. Prasad, and K. Toyama, "Optimal Audio-Visual Representations for Illiterate Users of Computers" in the procedings of 16th International conference on World Wide Web, Albarta, Canada, pp 873-882, 2007.

[28] I. Medhi, and K. Toyama, "Full-Context Videos for First-Time, NonLiterate PC Users," in the procedings of Information and Communication Technologies and Development, 2007, India, PP 1-9, November 2007. 
[29] A. Rahman, T. Nakanishi, A Fukuda, "Delay Tolerant Network for Developing Countries," in the Proceedings of International Conference on Informatics, Electronics \& Vision, Dhaka, Bangladesh, May 2013. 